\title{
Preparation of Organic-Inorganic Hybrid Materials Based on MCM-41 and Its Applications
}

\author{
Sana M. Alahmadi, ${ }^{1,2}$ Sharifah Mohamad, ${ }^{1}$ and Mohd Jamil Maah ${ }^{1}$ \\ ${ }^{1}$ Department of Chemistry, Faculty of Science, University of Malaya, 50603 Kuala Lumpur, Malaysia \\ ${ }^{2}$ Department of Chemistry, Faculty of Science, Taibah University, Almadina Almonawara 30001, Saudi Arabia
}

Correspondence should be addressed to Sana M. Alahmadi; so2002na@yahoo.com

Received 3 May 2013; Accepted 28 September 2013

Academic Editor: S. Komarneni

Copyright (C) 2013 Sana M. Alahmadi et al. This is an open access article distributed under the Creative Commons Attribution License, which permits unrestricted use, distribution, and reproduction in any medium, provided the original work is properly cited.

\begin{abstract}
This work reports the covalent attachment of three different calix[4] arenes (calix[4]arene (C4), p-sulfonatocalix[4]arene (C4S), and p-tert-butyl-calix[4]arene (PC4)) to MCM-41, using a three-step modification process. 3-Chloropropyltrimethoxysilane (ClPTS) was first attached to the mesoporous silica surface and subsequently converted to amides via the reaction with toluene diisocyanate (TDI). Finally, calix[4] arene derivatives attached to the isocyanate ending remained available on toluene di-iso-cyanate. Changes in the surface properties of the mesoporous silica caused by the chemical modification were monitored using the Fourier transform infrared spectroscopy (FTIR), thermal analysis (TGA), and elemental analysis. The FTIR spectra and TGA analysis verify that the calix[4]arene derivatives are covalently attached to the mesoporous silica. The preservation of the MCM- 41 channel system was checked by the X-ray diffraction and nitrogen adsorption analysis. These materials were then used to evaluate the sorption properties of some organotins compounds (Tributyltin (TBT), Triphenyltin (TPT), and Dibutyltin (DBT)).
\end{abstract}

\section{Introduction}

The term "calixarene", proposed by David Gutsche in 1975, stems from the Greek word "calix" which means "vase" or "chalise" or "arene," indicating the existence of aryl rings. Calixarenes are defined as a class of cyclooligomers having distinct upper and lower rims and central annulus $[1,2]$.

Currently, calixarenes are also known as the third generation supramolecules after cyclodextrins and crown ethers [3]. Calixarenes chemistry is a curious combination of academic and industrial research. The varying calixarene applications are attributed to their cup-like shape with structured upper and lower rims and central annulus [1] selective complexation with several metal ions and neutral molecules, stability, solubility, and their large scale availability [4]. Calixarenes have commonly been utilized for the purpose of separation [5] and extraction of various analytes such as the selective removal of aromatic amines [6], heavy metal [7-9], dyes [10$12]$, and oxyanions $[13,14]$. They have also been utilized in several chemical sensor devices including ion and moleculeselective electrodes for groups of ions and molecules $[15,16]$, fluorescent sensors $[17,18]$, and nonlinear optical sensors [19]. The immobilization of calixarenes within organic or inorganic matrices makes it possible to take advantage of their unique properties in the solid state.

Mesoporous silica is the most commonly utilized support material for the production of functionalized materials, owing to their great physical strength and chemical inertness [20-28]. A variety of coupling agents have also been utilized to functionalize mesoporous silica like organosilanes with particular functional groups, for instance, chloride, carboxylic acid, thiol, and amine [29]. Isocyanates are considered to be highly reactive with $-\mathrm{OH}$ groups, leading to the formation of urethane bonds [30, 31], which depend on the utilized diisocyanate, at least as strong as those obtained with the organosilane binders [31]. The preparation of mesoporous silica functionalized by calix[4] arene (C4), psulfonatocalix[4] arene (C4S), and p-tert-butyl-calix[4] arene (PC4) using toluene diisocyanate as a linker was recently reported for the first time in our previous work [32].

Organotins (OTs) compounds are invaluable organometallic pollutants which are utilized as follows: as antifouling 
paints, PVC stabilizers, pesticides, and additives in countless industrial, chemical, and agricultural applications [33, 34]. The increasing use of tin compounds in the last few years has led to a high degree of organotins compounds widely detected in environmental matrices [35]. Additionally, the high toxicity of the organotins compounds raised, poses increasing environmental concerns. Hence, in several countries, the utilization of triorganotin compounds including TBT and TPT is legally banned or restricted. The adsorption method has been viewed as among the most effective and economical ways to remove organic and inorganic pollutants from aqueous solutions [36].

In this work, mesoporous silica MCM-41 was functionalized with calix[4] arene derivatives by postgrafting methods using a linking agent consisting of an organosilane (3chloropropyltrimethoxysilane-ClPTS) and a toluene 2,4-diiso-cyanate (TDI) as new materials. In this method, one of the isocyanate endings at TDI is attached to the organosilane -OH ending, while the other isocyanate ending remains available for reaction with calix[4] arene derivatives.

Based on our knowledge, no research result on the mesoporous silica MCM-41 functionalized with calix[4] arene derivatives for the OTs removal has been reported in the literature. Therefore, the objectives of this research are to synthesize and characterize mesoporous silica MCM-41 functionalized with calix[4]arene derivatives and study the effectiveness of this material as adsorbent for the removal of OTs.

\section{Experimental}

2.1. Materials. The chemicals used in this are commercially available. Mesoporous silica Aldrich, surface area $993 \mathrm{~m}^{2} / \mathrm{g}$, average diameter of $2.9 \mathrm{~nm}$ serves as silica sources and calix[4] arene (Acros) and p-tert-butylcalix[4]arene (Fluka) as the organic modifier. 3-Chloropropyltriethoxysilane (ClPTS) (Aldrich) and toluene 2,4-di-iso-cyanate (TDI) (Aldrich) were the organic linkers. Triethylamine SAFC was used as catalyst. Di-n-butylamine Acros and hydrochloric acid Fisher were used for the determination of isocyanate groups. Toluene Fisher was dried before use by using molecular sieves, whereas ethanol Fisher and acetone Fisher were used as solvents. Dichloromethane (Sigma Aldrich), chlorosulfonic acid (Merk), and methanol were useful for the synthesis of p-sulfonatocalix[4] arene as described in the literature [37]. Water was purified using Milli-Q purification equipment.

For sorption experiments, tributyltin chloride (Aldrich), triphenyltin chloride (Fluka), and dibutyltin chloride (Aldrich) concentrations were adjusted by successive dilutions with Milli-Q water of an $8.42 \mathrm{mM}$ solutions in methanol stored at $4^{\circ} \mathrm{C}$ in the dark.

2.2. Instrumentation. Fourier transform infrared spectra (FTIR) were recorded on a Perkin Elmer FTIR Spectrum RX1 ATR with a $\mathrm{KBr}$ pellet technique. Thermogravimetry (TG) and differential thermal analysis (DTA) were carried out from 50 to $900^{\circ} \mathrm{C}$ at a heating rate of $20^{\circ} \mathrm{C} / \mathrm{min}$ in a nitrogen
TABLE 1: ICP-MS conditions.

\begin{tabular}{lc}
\hline Parameter & Value \\
\hline RF power $(\mathrm{W})$ & 1550 \\
Sampling depth $(\mathrm{mm})$ & 8 \\
Carrier gas flow $(\mathrm{L} / \mathrm{min})$ & 0.9 \\
Make-up gas flow $(\mathrm{L} / \mathrm{min})$ & 0.28 \\
$\mathrm{O}_{2} /$ Ar mixed gas & 0 \\
Chamber temperature & $2^{\circ} \mathrm{C}$ \\
Nebuliser & Babington \\
Cones & $\mathrm{Ni}$ \\
\hline
\end{tabular}

atmosphere using Perkin Elmer TGA 4000 analyzer. Elemental analyses were performed on a Perkin Elmer CHNS2400 analyzer. Nitrogen adsorption-desorption experiments were carried out at $77.40 \mathrm{~K}$ on a Quantachrome Autosorb Automated Gas Sorption system. The Brunauer-EmmettTeller (BET) surface area (SBET) was calculated from the linearity of the BET equation. The volume and pore diameter were calculated from the pore size distribution curves using the Density Functional (Theory) (DFT) method. The X-ray powder diffraction (XRD) patterns were obtained on a Bruker AXS D-8 Advance diffractometer using $\mathrm{Cu} \mathrm{K}$ radiation $(\lambda=$ $0.154056 \mathrm{~nm}$ ) at $40 \mathrm{kV}$ and $30 \mathrm{~mA}$ within the $2 \theta$ range of 2 to 10 .

An Agilent Technology 7500 series ICP-MS was used for the determination of OTs in aqueous solutions. The ICPMS condition was shown in Table 1. A series of Sn standard solutions from 0 to $1000 \mu \mathrm{g} / \mathrm{L}$ were used to construct the calibration curve, on which a good linear relationship was observed.

\subsection{Synthesis Methods}

2.3.1. Preparation of 3-Hydroxypropyl Trimethylsilyl Functionalized MCM-41. The surface functionalization of MCM-41 was carried out by a procedure described by Feng et al. [38]. The functionalization of the surface of MCM-41 was performed according to Figure 1. The MCM-41 support was dehydrated $\left(125^{\circ} \mathrm{C}\right.$, overnight) and a self-assembled monolayer of the initial silane was produced by suspending $1.0 \mathrm{~g}$ of MCM-41 in $40 \mathrm{~mL}$ of toluene in a $100 \mathrm{~mL}$ round bottom flask. The suspension was stirred vigorously for 5 minutes before adding $0.30 \mathrm{~mL}$ of DI water and the stirring continued for two hours. A slight excess of the silane $(10 \% \mathrm{v} / \mathrm{v}), 2.18 \mathrm{~mL}$ of 3-chloropropyltriethoxysilane corresponding to $9.07 \mathrm{mmol}$, was added and the solution refluxed for 6 hours. The solids are then filtered and washed copiously with toluene and acetone to remove unreacted silane and dried overnight. The modified material obtained is denoted as CIPTS-MCM. The chlorine groups present in the CIPTS-MCM were hydrolysed into hydroxyl groups by heating at $60^{\circ} \mathrm{C}, 1.0 \mathrm{~g}$ of the solid material with a solution of methanol: water $(1: 1)$ for two hours [29]. The hydrolysed material (OHPTS-MCM) was filtered and dried at $110^{\circ} \mathrm{C}$ overnight.

The hydrolysed material (OHPTS-MCM) was refluxed with the excess of TDI (dried by molecular sieve for $24 \mathrm{~h}$ ) in 


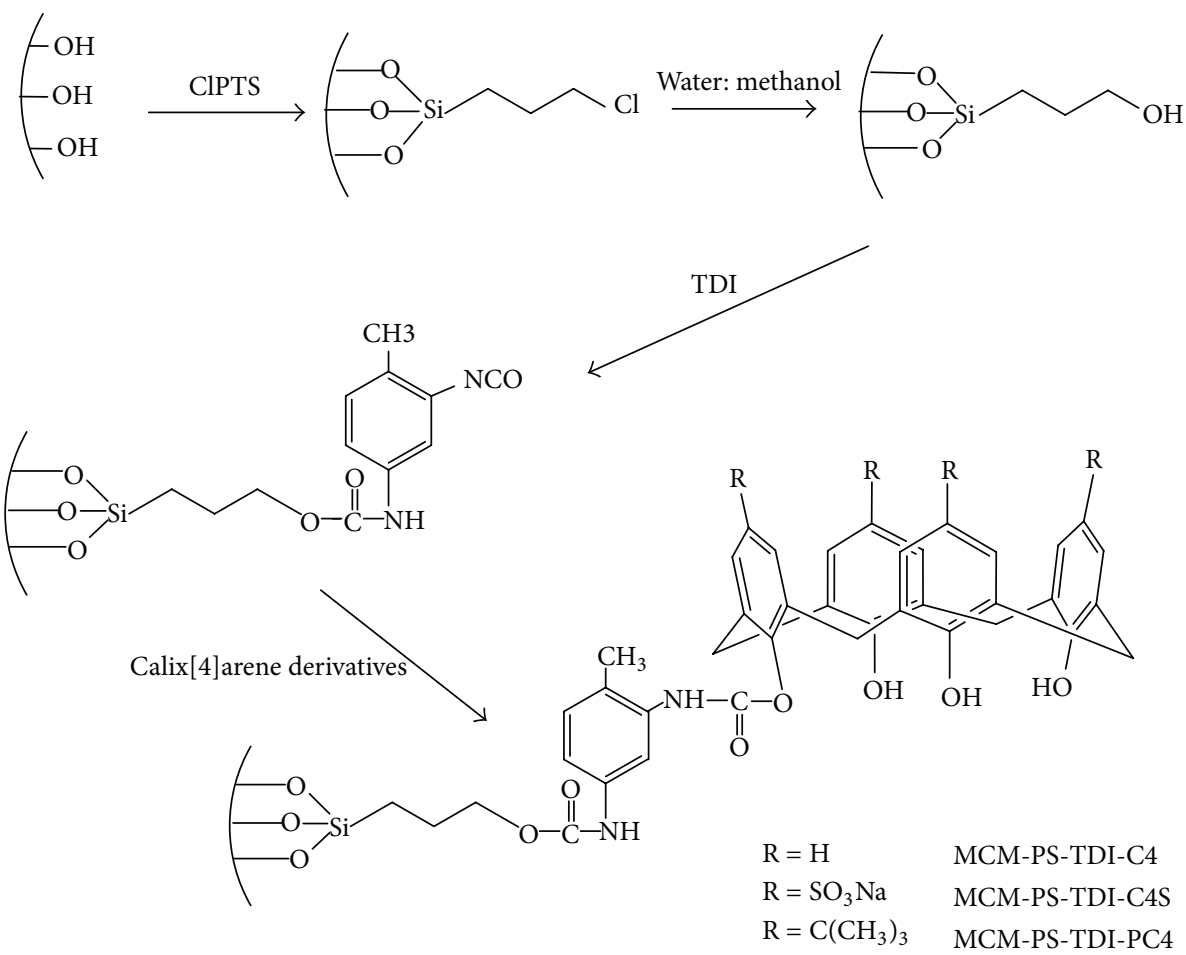

FIGURE 1: Preparation of modified mesoporous silica with calix[4] arene derivatives.

a dry nitrogen atmosphere at $80^{\circ} \mathrm{C}$ for $4 \mathrm{~h}$ [39]. After cooling down, the material was filtered, washed, and dried overnight and denoted as MCM-PS-TDI.

2.3.2. Immobilizing of Calix[4]arene Derivatives onto MCMPS-TDI. Mesoporous silica-supported calix[4] arene derivatives were prepared by refluxing $1.0 \mathrm{~g}$ of the functionalized mesoporous silica (MCM-PS-TDI) with $1.8 \mathrm{mmol}$ of calix[4] arene derivatives (calculated from Section 2.3.3) and a few drops of triethylamine in $100 \mathrm{~mL}$ of dry toluene in a 250-mL round-bottom flask and the reaction temperature was kept at $80^{\circ} \mathrm{C}$ for $24 \mathrm{~h}$ under stirring. The modified materials were filtered, washed by toluene and acetone, and dried under vacuum. The samples were marked as MCM-PS-TDI-C4 for calix[4] arene, MCM-PS-TDI-C4S for p-sulfonatocalix[4] arene, and MCM-PS-TDI-PC4 for p-tertbutyl-calix[4]arene.

2.3.3. Determination of Isocyanate Groups. The content of isocyanate groups of the reaction system was determined by titration. $200 \mathrm{mg}$ of MCM-PS-TDI sample and $20 \mathrm{~mL}$ of $0.1 \mathrm{~mol} \mathrm{~L}^{-1}$ di-n-butylamine in toluene were charged into a flask and the mixture was stirred at room temperature for $1 \mathrm{~h}$. The unreacted di-n-butylamine was back titrated with $0.1 \mathrm{~mol} \mathrm{~L}^{-1} \mathrm{HCl}$ using bromophenol blue as an indicator. The content of isocyanate groups was calculated by:

$$
\text { isocyanate group }\left(\mathrm{mmolg}^{-1}\right)=0.1\left(V_{o}-V_{s}\right) \frac{f}{w}
$$

where $V_{o}(\mathrm{~mL})$ is the titer of $0.1 \mathrm{~mol} \mathrm{~L}^{-1} \mathrm{HCl}$ for blank; $V_{s}$ $(\mathrm{mL})$ is the titer of $0.1 \mathrm{~mol} \mathrm{~L}^{-1} \mathrm{HCl}$ for the sample; $f$ is the factor of $0.1 \mathrm{~mol} \mathrm{~L}^{-1} \mathrm{HCl}$; and $w$ is the weight of the sample in $\operatorname{gram}(\mathrm{g})$.

2.4. Sorption Studies. Mesoporous silica-grafted calix[4]arene derivatives $(0.01 \mathrm{~g})$ were shaken with $10 \mathrm{~mL}$ of aqueous solution containing OTs solution with known concentration $\left(C_{o}\right)$ at $180 \mathrm{rpm}$ in $50 \mathrm{~mL}$ Teflon reactors (FEP, Nalgene) since other studies have shown that this material does not compete for OTs sorption and does not leach OTs compounds [40]. Batch experiments were performed at room temperature for $1 \mathrm{~h}$. The adsorbent had been removed by filtration and the concentration of OTs remaining $\left(C_{e}\right)$ in the aqueous phase after the sorption was then determined by ICP-MS. The percentage of sorption of OTs $(S \%)$ has been calculated as

$$
S \%=\left[\frac{C_{o}-C_{e}}{C_{o}}\right] \times 100,
$$

where $C_{o}$ is the initial aqueous concentration of OTs $\left(\mathrm{mg} \mathrm{L}^{-1}\right)$ and $C_{e}$ is the concentration of OTs after shaking for a certain period of time $\left(\mathrm{mg} \mathrm{L}^{-1}\right)$.

\section{Results and Discussion}

In this work, the MCM-41 was functionalized through a postsynthetic method, with 3-chloropropyltrimethoxysilane (ClPTS) [38]. The chlorine groups were hydrolysed into hydroxyl groups [30], which reacted with one of the terminal isocyanate groups of the linking agent (TDI), forming 
urethane links. The isocyanate groups at parapositions in TDI would bind with the hydroxyl groups on the surface of OHPTS-MCM preferentially, whereas those at the orthopositions would be preserved due to the steric hindrance within the TDI molecule [41]. The mole amount of the isocyanate groups that reacted with OHPTS-MCM can be regarded as that of TDI that reacted with OHPTS-MCM. The amounts of TDI that reacted with OHPTS-MCM are largely dependent on the amount of hydroxyls on the surface so in the case of the excess of TDI, the amounts of TDI that reacted with OHPTSMCM were invariable $[42,43]$. The isocyanate groups at orthopositions in the OHPTS-MCM functionalized with TDI had reacted with hydroxyl groups at calix[4]arene derivatives.

\subsection{Materials Characterization}

3.1.1. FTIR Analysis. The functionalization of MCM- 41 using CIPTS and TDI can be identified using FTIR. Figure 2 shows the FTIR spectra of unmodified mesoporous silica and functionalized mesoporous silica with ClPTS and TDI samples, MCM-41, and MCM-PS-TDI, respectively. The spectrum of the unmodified MCM-41 (Figure 2), as well as the modified materials, is dominated by strong bands which are characteristic of the support matrix, indicating that the support framework had remained unchanged. These bands are due to the surface hydroxyl groups, in the range of 3770$3300 \mathrm{~cm}^{-1}$, and due to lattice vibrations, in the range of $1300-750 \mathrm{~cm}^{-1}$. Bands at about $1215,1085,807$, and $480 \mathrm{~cm}^{-1}$ are assignable to the asymmetric and symmetric stretching $\left(v_{\text {as }}(\mathrm{Si}-\mathrm{O}-\mathrm{Si})\right.$ and $\left.v_{\mathrm{s}}(\mathrm{Si}-\mathrm{O}-\mathrm{Si})\right)$ of the support framework. The band present at about $970 \mathrm{~cm}^{-1}$ is attributable to $\nu$ (Si$\mathrm{OH})$ vibrations [44-48].

After the anchoring of 3-chloropropyltriethoxysilane CIPTS-MCM (not shown here), and the subsequent hydrolysis (OHPTS-MCM), new weak bands arise at 2960 and $2850 \mathrm{~cm}^{-1}$ probably due to the aliphatic $\left(-\mathrm{CH}_{2}\right)$ stretching of the propyl chain of the silylating agent, suggesting that the modification of the support material was achieved [47, 49].

The addition of excess TDI to the OHPTS-MCM resulted in the incorporation of isocyanate functionalities on the surface of the OHPTS-MCM (Figure 1). This was evidenced by the appearance of a clearly discernible band at $2282 \mathrm{~cm}^{-1}$ corresponding to the asymmetric stretching of the appended terminal isocyanate groups, and the appearance of an aromatic $\mathrm{C}-\mathrm{C}$ stretch at $1560 \mathrm{~cm}^{-1}$ in the FTIR spectrum. The signals corresponding to the $\mathrm{C}=\mathrm{O}$ and $\mathrm{C}-\mathrm{N}$ stretches of the carbamate linkages formed between the hydroxyl group and the isocyanate functionality at $1637 \mathrm{~cm}^{-1}$ and $1202 \mathrm{~cm}^{-1}$ had merged with the band of surface hydroxyl groups of mesoporous silica and $\mathrm{Si}-\mathrm{O}-\mathrm{Si}$ band, respectively.

The infrared spectra obtained for MCM-PS-TDI-C4, MCM-PS-TDI-C4S and MCM-PS-TDI-PC4 are shown in Figure 3. Typical silica bands associated with the main inorganic backbone can be clearly observed as a large broad band between 3400 and $3200 \mathrm{~cm}^{-1}$, attributed to the presence of the $\mathrm{O}-\mathrm{H}$ stretching frequency of silanol groups bonded to the inorganic structure, and the intense band related to the $\mathrm{Si}-\mathrm{O}-$ Si stretching of these groups at $1080-1225 \mathrm{~cm}^{-1}$. All spectra

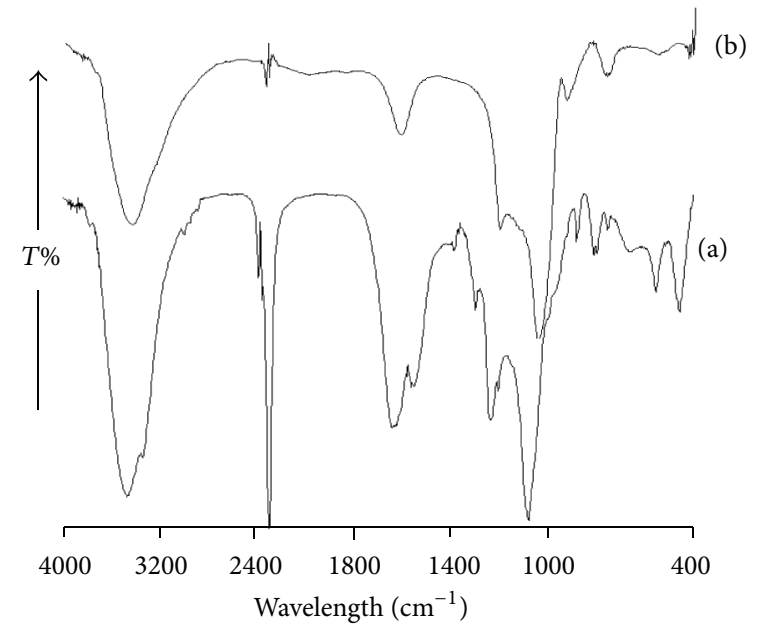

FIGURE 2: Fourier transform infrared spectroscopy (FTIR) spectra of MCM-PS-TDI (a) and MCM-41 (b).

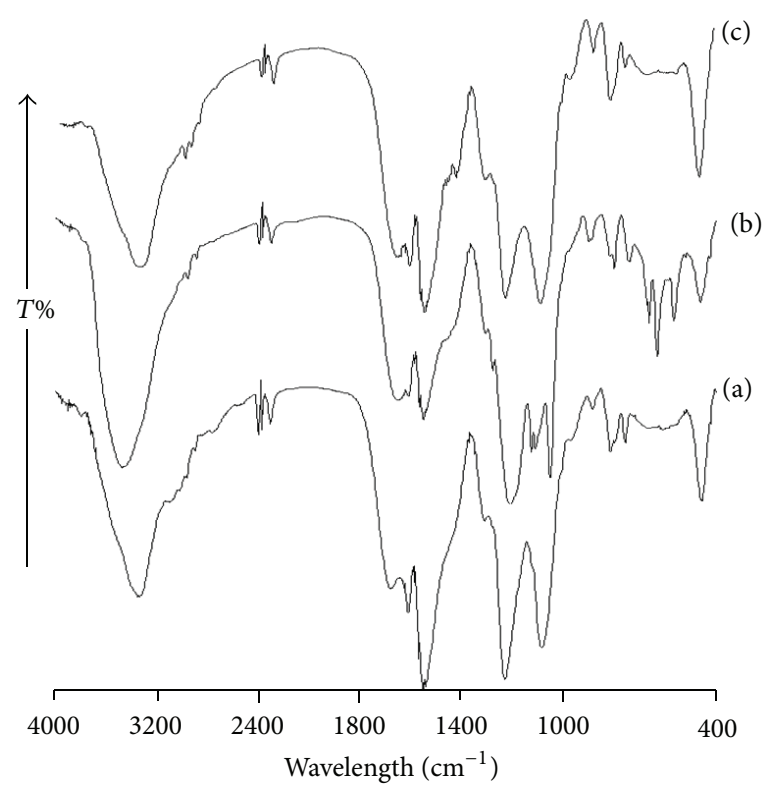

FIgURE 3: FTIR spectra of MCM-PS-TDI-C4 (a), MCM-PS-TDIC4S (b) and MCM-PS-TDI-PC4 (c).

show a large band around $1500-1650 \mathrm{~cm}^{-1}$ due to $\mathrm{C}_{\mathrm{ar}}-\mathrm{C}_{\mathrm{ar}}$ stretching. Additional bands are also observed in the spectra that confirm the presence of calix [4] arene derivatives in these materials. The bands at $1418 \mathrm{~cm}^{-1}$ and $1448 \mathrm{~cm}^{-1}$ of MCMPS-TDI-C4 spectrum (Figure 3(a)), which are referred to $\mathrm{C}_{\mathrm{ar}^{-}}{ }^{-}$ $\mathrm{OH}$ and methylene bridges $-\mathrm{CH}_{2}-$ groups, were broadened with $\mathrm{C}_{\mathrm{ar}}-\mathrm{C}_{\mathrm{ar}}$ stretching at $1541 \mathrm{~cm}^{-1}$. Based on these data, it is proven that the MCM-PS-TDI was successfully modified with calix[4]arene.

The spectrum of MCM-PS-TDI-C4S, (Figure 3(b)) had presented new peaks compared to MCM-PS-TDI. $\mathrm{C}_{\mathrm{ar}}-\mathrm{S}$ peaks and methylene bridges $-\mathrm{CH}_{2}-$ groups were confirmed by the strong absorptions at 662 and $630 \mathrm{~cm}^{-1}$ and $1443 \mathrm{~cm}^{-1}$, respectively. The strong absorption peaks for $\mathrm{SO}_{3}{ }^{-}$were 
present at 1048 and $1122 \mathrm{~cm}^{-1}$ [50]. These peaks validate the immobilization of the p-sulfonatocalix[4]arene to the isocyanate functional groups.

In the case of MCM-PS-TDI-PC4, Figure 3(c) generally shows a strong band at $1542 \mathrm{~cm}^{-1}$ and its shoulder near $1418 \mathrm{~cm}^{-1}$, which correspond to the phenyl $v \mathrm{C}_{\mathrm{ar}}-\mathrm{C}_{\mathrm{ar}}$ and methylene bridges $-\mathrm{CH}_{2}-$., respectively, and methyl $\left(\mathrm{CH}_{3}\right)$ asymmetric stretching and symmetric vibrations at 2969 and $2862 \mathrm{~cm}^{-1}$, respectively.

Meanwhile, the disappearance of the absorption peak of isocyanate group at 2280 indicates that the reserved isocyano groups had reacted with calix[4] arene derivatives.

3.1.2. Thermogravimetric Analysis. The thermogravimetric analysis of functionalized mesoporous silica MCM-PSTDI with calix[4]arene derivatives was also determined (Figure 4). The functionalized MCM-PS-TDI with calix[4]arene derivatives exhibited a weight loss at about $50^{\circ} \mathrm{C}$ corresponding to the loss of physically adsorbed water. With the increase in temperature, there is a significant change in the weight loss curve with functionalized MCM-PS-TDI. The removal of the organic moiety started at $180-240^{\circ} \mathrm{C}$ and continued up to 400 and $600^{\circ} \mathrm{C}$. The sharp decrease in weight at $280^{\circ} \mathrm{C}$ is due to the loss of the carbamate group (Table 2).

3.1.3. Elemental Analysis. The elemental analysis of the samples (Table 3 ) shows a gradual increase in the carbon content after the modification step.

After the reaction of OHPTS-MCM with TDI, the percentages of $\mathrm{C}$ and $\mathrm{N}$ increase from $3.13 \%$ and $0.14 \%$ to $20.82 \%$ and $5.03 \%$, respectively, implying the success of the modification step. After reacting with C4, C4S, and PC4 the C content increased further to $30.93 \%, 25.83 \%$, and $33.63 \%$, respectively, showing that the calix[4] arene derivatives were attached to the silicate surface.

3.1.4. XRD Diffraction. The functionalized ordered mesoporous material, MCM-PS-TDI-C4, MCM-PS-TDI-C4S, and MCM-PS-TDI-PC4 were characterized by XRD. The diffraction patterns are shown in Figure 5. The XRD patterns of the samples show weak (100) peaks and weaker (110) and (200) peaks. The (100) peak gradually shifts to higher angles and the loss of the peak intensity, indicates the presence of the organic moieties on the internal pore walls of MCM-41 [51]. The peaks (110) and (200) point to a decrease in the overall intensities of XRD reflections of MCM-41 after calix[4] arene derivatives functionalization (Figure 5). This may be due to the difference of the scattering contrast between the amorphous silicate framework and organic moieties, which are located inside the channels of MCM-41 [52, 53].

3.1.5. Nitrogen Adsorption-Desorption Analysis. Nitrogen adsorption-desorption experiments yielded BrunauerEmmett-Teller (BET) surface area of $993 \mathrm{~m}^{2} / \mathrm{g}$ for MCM-41 and total pore volumes of 0.86 . After the reaction with the coupling agent and modification with the calix[4]arene

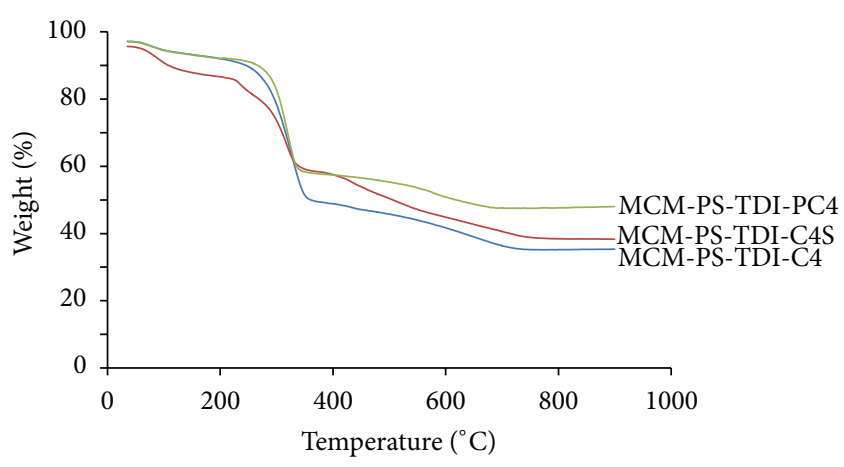

FIGURE 4: TGA analysis of MCM-PS-TDI-C4, MCM-PS-TDI-C4S, and MCM-PS-TDI-PC4.

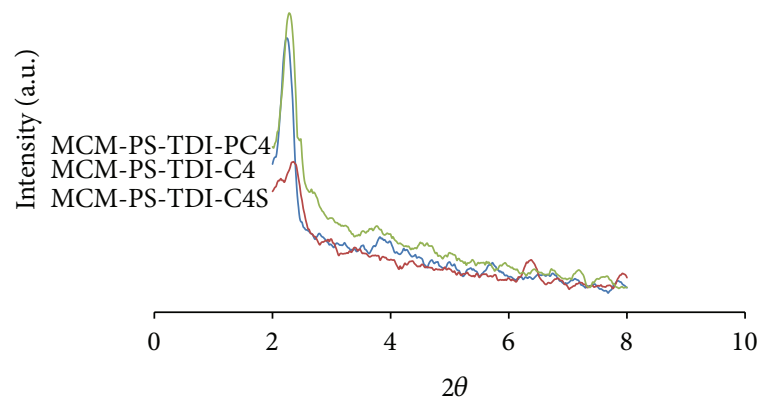

FIGURE 5: XRD analysis of MCM-PS-TDI-C4, MCM-PS-TDI-C4S, and MCM-PS-TDI-PC4.

derivatives, both the surface area and the total pore volume had dropped significantly (Table 4 ).

Figure 6 shows the nitrogen adsorption-desorption isotherms for the mesoporous silicates. All curves presented a Type-II isotherm, characteristic of nonporous or macroporous adsorbent with strong adsorbate-adsorbent interactions. Adsorption isotherms are of Type II in the IUPAC classification that represents monolayer/multilayer adsorption. This suggests that the adsorption of $\mathrm{N}_{2}$ for the sorbent materials is moderate or, on the other hand, that the main adsorption process may be ascribed to a van der Waals force. Generally, the isotherm shows that the amount of $\mathrm{N}_{2}$ adsorbed increases as the relative pressure increases up to a saturation point. A complete adsorption of $\mathrm{N}_{2}$ as a monolayer onto the surface of the sorbent material is shown by the plateau of the adsorption isotherm. After this point, a large uptake of $\mathrm{N}_{2}$ is observed to be close to the saturation pressure and it is assumed that a multilayer adsorption has taken place (i.e., implying the presence of mesoporous) [54].

The grafted materials exhibit a broader pore diameter and display a decrease in the surface area and pore volume (Table 4). The decrease of the pore value and the broad distribution of pore size prove that the calix[4] arene derivatives in the grafted mesoporous samples are mainly located on the internal surfaces of the mesoporous materials $[47,52,55]$.

3.2. Evaluation of Sorption Capabilities. This study also aims to evaluate the sorption abilities by mesoporous silica grafted 
TABLE 2: Results of thermogravimetric analysis.

\begin{tabular}{lccc}
\hline Sample & Region ${ }^{\circ} \mathrm{C}$ & Weight loss $\%$ & Assignment \\
\hline & $45-120$ & 3.4 & Moisture \\
MCM-PS-TDI-C4 & $120-200$ & 2.4 & Calix[4] arene \\
& $200-350$ & 42.1 & Linkers and calix [4]arene \\
& $350-800$ & 14.1 & Calix[4] arene \\
\hline \multirow{3}{*}{ MCM-PS-TDI-C4S } & $45-150$ & 8.3 & Moisture \\
& $280-350$ & 21.8 & Linkers \\
& $350-800$ & 19.8 & Calix[4]arene sulfonate \\
MCM-PS-TDI-PC4 & $45-150$ & 3.3 & Moisture \\
& $150-350$ & 34.5 & Linkers and paratert butyl Calix[4] arene \\
& $400-700$ & 10.4 & Paratert butyl calix[4]arene \\
\hline
\end{tabular}

TABLE 3: Results of elemental analysis for MCM-PS-TDI functionalized with calix[4] arene derivatives.

\begin{tabular}{lcccc}
\hline Sample & $\% \mathrm{C}$ & $\% \mathrm{H}$ & $\% \mathrm{~N}$ & $\% \mathrm{~S}$ \\
\hline MCM-PS-TDI-C4 & 30.93 & 3.62 & 5.21 & - \\
MCM-PS-TDI-C4S & 25.83 & 3.23 & 2.11 & 3.71 \\
MCM-PS-TDI-PC4 & 33.63 & 3.97 & 4.46 & - \\
\hline
\end{tabular}

TABLE 4: Structural parameters of MCM-41, MCM-PS-TDI-C4, MCM-PS-TDI-C4S, and MCM-PS-TDI-PC4.

\begin{tabular}{lccc}
\hline Sample & $S_{\mathrm{BET}}\left(\mathrm{m}^{2} / \mathrm{g}\right)$ & $V\left(\mathrm{~cm}^{3} / \mathrm{g}\right)$ & $D(\mathrm{~nm})$ \\
\hline MCM-41 & 993 & 0.86 & 2.9 \\
MCM-PS-TDI-C4 & 32.1 & 0.45 & 11.8 \\
MCM-PS-TDI-C4S & 30.6 & 0.38 & 9.7 \\
MCM-PS-TDI-PC4 & 10.4 & 0.11 & 22.1 \\
\hline
\end{tabular}

with calix[4] arene derivatives toward some selected organotin compounds. We have performed several solid-liquid sorption studies of organotins compounds (Tributyltin TBT, Triphenyltin TPT, and Dibutyltin DBT) by using MCM-PSTDI-calix[4] arene derivatives as sorbents. The sorption percentages were calculated in (2) and they are given in Figure 7. These data have been obtained by using a $0.01 \mathrm{~g}$ of the grafted mesoporous silica for the sorption of organotins compounds from aqueous solution. The equilibrium concentration of tin in the aqueous phase has been determined by ICP-MS (Table 1). The sorption data reveal that MCM-PS-TDI-C4S was the most efficient sorbent.

The existence of calix[4]arenes on the surface of the adsorbent plays an important role in the adsorption process. Calix[4]arenes are able to bind some cations, anions, and neutral molecules to form inclusion complexes in aqueous solution. Extensive studies of molecular recognition by calixarenes have revealed that the size/shape-fit concept plays a crucial role in the formation of the inclusion complexes of host compounds with guest molecules of various structures. Therefore, weak intermolecular forces such as ion-dipole, dipole-dipole, dipole-induced dipole, van der Waals, electrostatic interaction, hydrogen bonding, and hydrophobic interaction ( $\mathrm{CH}-\pi$ or $\pi-\pi$ ) are known to contribute cooperatively to the inclusion complexation of guest molecules

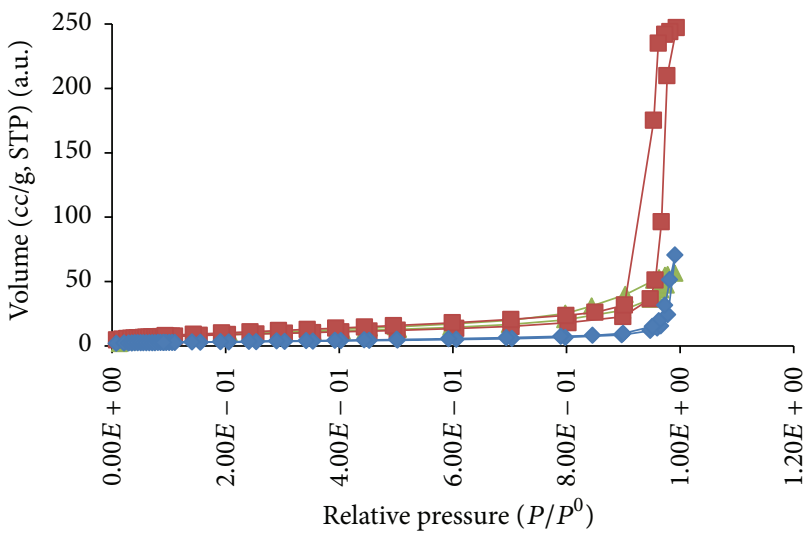

FIGURE 6: Nitrogen adsorption-desorption isotherms of MCM-PSTDI-C4 ( $\Delta$ ), MCM-PS-TDI-C4S ( $\square)$, and MCM-PS-TDI-PC4 ( $)$ ).

with calixarenes according to the size/shape-fit concept. In the present case, the van der Waals forces and hydrophobic interaction are considered to determine the complex stability to a large extent. Moreover, the electrostatic interaction is also considered in case of MCM-PS-TDI-C4S. High adsorption capacity for MCM-PS-TDI-PC4 may attribute to the higher value of the pore diameter.

\section{Conclusion}

In this work, we report the successful anchoring of calix[4]arene derivatives onto the MCM-41 surface, using 3-chloropropyltriethoxysilane (CIPTS). The chlorine groups were hydrolysed into hydroxyl groups, which reacted with one of the terminal isocyanate groups of the linking agent (TDI), forming urethane links. The other terminal isocyanate functionality reacts with the free hydroxyl group present in the calix[4]arene derivatives. Different characterization techniques such as FTIR, XRD, CHN, BET, and Thermogravimetric analysis have shown evidence that the calix[4] arene derivatives were covalently attached to the MCM-41 surface and that their channel structure had remained unchanged. The sorption studies of some organotins compounds were performed by MCM-PSTDI-calix[4]arene derivatives sorbent materials. From 


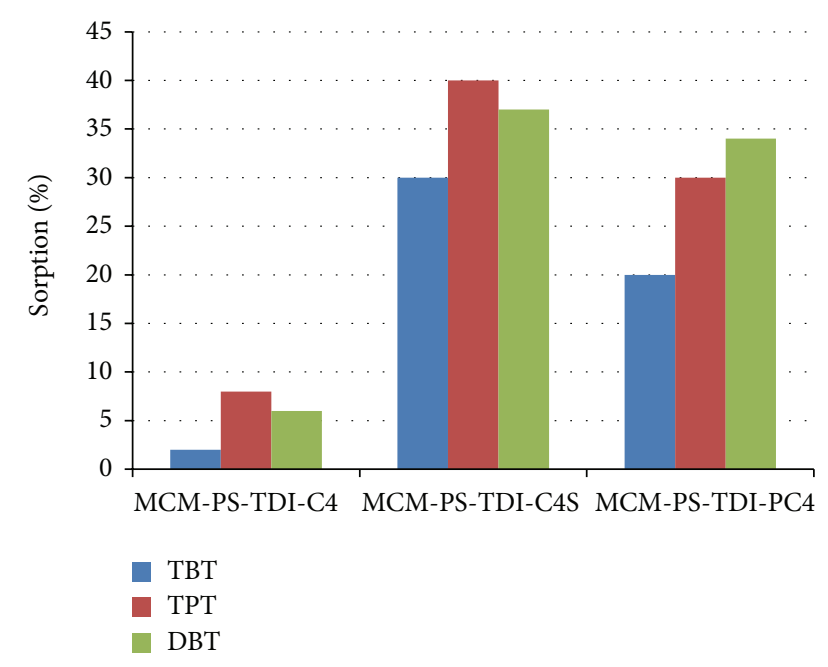

FIGURE 7: Sorption percentages of organotins compounds by MCMPS-TDI-calix[4] arene derivatives sorbent.

the sorption results, it appeared that MCM-PS-TDI-C4S was the most efficient sorbent.

\section{Acknowledgments}

This work was supported by the University of Malaya Research Grant (Project no. RG169/12SUS) and the High Impact Research Ministry of Higher Education (HIR MoHE (faculty) F0004-21001), University of Malaya. Taibah University is also acknowledged for financial support.

\section{References}

[1] D. R. Stewart and C. D. Gutsche, "Isolation, characterization, and conformational characteristics of p-tert-Butylcalix[9-20] arenes ", Journal of the American Chemical Society, vol. 121, pp. 4136-4146, 1999.

[2] C. D. Gutsche, Calixarenes: A Versatile Class of Macrocyclic Compounds, Kluwer Academic, Dodrecht, The Netherlands, 1991.

[3] C. D. Gutsche, Calixarenes: An Introduction, RSC Publishing, 2nd edition, 2008.

[4] R. M. Izatt, J. J. Christensen, and R. T. Hawkins, "Recovery of cesium," U.S. Patent. 4, 477, 1984.

[5] G. Arena, A. Casnati, A. Contino, L. Mirone, D. Sciotto, and R. Ungaro, "Synthesis of new calixcrowns and their anchoring to silica gel for the selective separation of $\mathrm{Cs}^{+}$and $\mathrm{K}^{+}$," Chemical Communications, no. 19, pp. 2277-2278, 1996.

[6] T. Aksoy, S. Erdemir, H. B. Yildiz, and M. Yilmaz, "Novel watersoluble calix[4,6]arene appended magnetic nanoparticles for the removal of the carcinogenic aromatic amines," Water, Air and Soil Pollution, vol. 223, pp. 4129-4139, 2012.

[7] I. H. Gubbuk, L. Gürfidan, S. Erdemir, and M. Yilmaz, "Surface modification of sporopollenin with calixarene derivative," Water, Air and Soil Pollution, vol. 223, pp. 2623-2632, 2012.

[8] M. Tabakci, S. Erdemir, and M. Yilmaz, "Preparation, characterization of cellulose-grafted with calix[4] arene polymers for the adsorption of heavy metals and dichromate anions," Journal of Hazardous Materials, vol. 148, no. 1-2, pp. 428-435, 2007.
[9] B. B. Adhikari, M. Kanemitsu, H. Kawakita, J. Jumina, and K. Ohto, "Synthesis and application of a highly efficient polyvinylcalix[4] arene tetraacetic acid resin for adsorptive removal of lead from aqueous solutions," Chemical Engineering Journal, vol. 172, no. 1, pp. 341-353, 2011.

[10] M. A. Kamboh, I. B. Solangi, S. T. H. Sherazi, and S. Memon, "A highly efficient calix[4] arene based resin for the removal of azo dyes," Desalination, vol. 268, no. 1-3, pp. 83-89, 2011.

[11] M. A. Kamboh, B. Solangi, S. T. H. Sherazi, and S. Memon, "Sorption of congo red onto p-tert-butylcalix[4]arene based silica resin," Journal of the Iranian Chemical Society, vol. 8, no. 1, pp. 272-279, 2011.

[12] M. A. Kamboh, I. B. Solangi, S. T. H. Sherazi, and S. Memon, "Synthesis and application of calix[4]arene based resin for the removal of azo dyes," Journal of Hazardous Materials, vol. 172, no. 1, pp. 234-239, 2009.

[13] S. Sayin, F. Ozcan, S. Memon, and M. Yilmaz, "Synthesis and oxoanions (dichromate/arsenate) sorption study of $\mathrm{N}$-methylglucamine derivative of calix[4]arene immobilized onto poly[(phenyl glycidyl ether)-co-formaldehyde]," Journal of Inclusion Phenomena and Macrocyclic Chemistry, vol. 67, pp. 385-391, 2010.

[14] S. Sayin, F. Ozcan, and M. Yilmaz, "Preparation and application of calix[4]arene derivatives bearing pyridinium units-grafted magnetite nanoparticles for removal of dichromate and arsenate anions," Journal of Macromolecular Science A, vol. 48, pp. 365372, 2011.

[15] V. K. Gupta, R. Ludwig, and S. Agarwal, "Anion recognition through modified calixarenes: a highly selective sensor for monohydrogen phosphate," Analytica Chimica Acta, vol. 538, pp. 213-218, 2005.

[16] P. A. Gale, Z. Chen, M. G. B. Drew, J. A. Heath, and P. D. Beer, "Lower-rim ferrocenyl substituted calixarenes: new electrochemical sensors for anions," Polyhedron, vol. 17, no. 4, pp. 405-412, 1998.

[17] M. Narita, Y. Higuchi, F. Hamada, and H. Kumagai, "Metal sensor of water soluble dansyl-modified thiacalix[4]arenes," Tetrahedron Letters, vol. 39, no. 47, pp. 8687-8690, 1998.

[18] H. Liu, Y. Xu, B. Li, G. Yin, and Z. Xu, "A new highly selective calix[4] arene-based fluorescent probe for $\mathrm{Ca}^{2+}$," Chemical Physics Letters, vol. 345, pp. 395-399, 2001.

[19] M. Regayeg, F. Vocanson, A. Duport et al., "New calixarenes for nonlinear optics: synthesis and conformation of functionalized p-nitrocalix [4] arenes," Materials Science and Engineering C, vol. 21, no. 1-2, pp. 131-135, 2002.

[20] A. Bibby and L. Mercier, "Adsorption and separation of watersoluble aromatic molecules by cyclodextrin-functionalized mesoporous silica," Green Chemistry, vol. 5, no. 1, pp. 15-19, 2003.

[21] C. Liu, J. B. Lambert, and L. Fu, "A novel family of ordered, mesoporous inorganic/organic hybrid polymers containing covalently and multiply bound microporous organic hosts," Journal of the American Chemical Society, vol. 125, pp. 64526461, 2003.

[22] C. Liu, J. B. Lambert, and L. Fu, "Simple surfactant-free route to mesoporous organic-inorganic hybrid silicas containing covalently bound cyclodextrins," The Journal of Organic Chemistry, vol. 69, pp. 2213-2216, 2004.

[23] C. Park, K. Lee, and C. Kim, "Photoresponsive cyclodextrincovered nanocontainers and their sol-gel transition induced by molecular recognition," Angewandte Chemie International Edition, vol. 48, pp. 1275-1278, 2009. 
[24] H. Kim, S. Kim, C. Park, H. Lee, H. J. Park, and C. Kim, "Glutathione-induced intracellular release of guests from mesoporous silica nanocontainers with cyclodextrin gatekeepers," Advanced Materials, vol. 22, no. 38, pp. 4280-4283, 2010.

[25] R. Huq, L. Mercier, and P. J. Kooyman, "Incorporation of cyclodextrin into mesostructured silica," Chemistry of Materials, vol. 13, no. 12, pp. 4512-4519, 2001.

[26] W. Guo, J. Wang, S. J. Lee, F. Dong, S. S. Park, and C. S. Ha, "A general pH-responsive supramolecular nanovalve based on mesoporous organosilica hollow nanospheres," Chemistry, vol. 16, pp. 8641-8646, 2010.

[27] Y. Q. Feng, M. J. Xie, and S. L. Da, "Preparation and characterization of an L-tyrosine-derivatized $\beta$-cyclodextrin-bonded silica stationary phase for liquid chromatography," Analytica Chimica Acta, vol. 403, pp. 187-195, 2000.

[28] S. D. Bhat, B. V. K. Naidu, G. V. Shanbhag, S. B. Halligudi, M. Sairam, and T. M. Aminabhavi, "Mesoporous molecular sieve (MCM-41)-filled sodium alginate hybrid nanocomposite membranes for pervaporation separation of water-isopropanol mixtures," Separation and Purification Technology, vol. 49, no. 1, pp. 56-63, 2006.

[29] S. Alahmadi, "Modification of mesoporous silica MCM-41 and its applications-a review," Oriental Journal of Chemistry, vol. 28, pp. 1-11, 2012.

[30] H. Xia and M. Song, "Preparation and characterisation of polyurethane grafted single-walled carbon nanotubes and derived polyurethane nanocomposites," Journal of Materials Chemistry, vol. 16, pp. 1843-1851, 2006.

[31] Y. S. Chun, K. Ha, Y. J. Lee et al., "Diisocyanates as novel molecular binders for monolayer assembly of zeolite crystals on glass," Chemical Communications, no. 17, pp. 1846-1847, 2002.

[32] S. M. Alahmadi, S. Mohamad, and M. J. Maah, "Synthesis and characterization of mesoporous silica functionalized with calix[4]arene derivatives," International Journal of Molecular Sciences, vol. 13, pp. 13726-13736, 2012.

[33] C. J. Evans and S. Karpel, Distributors For the U.S. and Canada, Elsevier, New York, NY, USA, 1985.

[34] S. M. Alahmadi, "Organotin speciation analysis based on liquid or gas chromatography," Asian Journal of Chemistry, vol. 23, no. 9, pp. 3787-3791, 2011.

[35] G. B. Jiang, J. Y. Liu, and Q. F. Zhou, "Search for the contamination source of butyltin compounds in wine: agglomerated cork stoppers," Environmental Science and Technology, vol. 38, pp. 4349-4352, 2004.

[36] M. F. F. Sze, V. K. C. Lee, and G. McKay, "Simplified fixed bed column model for adsorption of organic pollutants using tapered activated carbon columns," Desalination, vol. 218, no. 1-3, pp. 323-333, 2008.

[37] M. Makha and C. L. Raston, "Direct synthesis of calixarenes with extended arms: p-phenylcalix $[4,5,6,8]$ arenes and their water-soluble sulfonated derivatives," Tetrahedron Letters, vol. 42, no. 35, pp. 6215-6217, 2001.

[38] X. Feng, G. E. Fryxell, L.-Q. Wang, A. Y. Kim, J. Liu, and K. M. Kemner, "Functionalized monolayers on ordered mesoporous supports," Science, vol. 276, no. 5314, pp. 923-926, 1997.

[39] M. Yang, Y. Gao, J. He, and M. Li, "Preparation of polyamide 6/silica nanocomposites from silica surface initiated ringopening anionic polymerization," EXPRESS Polymer Letters, vol. 1, pp. 433-442, 2007.

[40] P. Behra, É. Lecarme-Théobald, M. T. Bueno, and J.-J. Ehrhardt, "Sorption of tributyltin onto a natural quartz sand," Journal of Colloid and Interface Science, vol. 263, no. 1, pp. 4-12, 2003.
[41] R. G. Arnold, J. A. Nelson, and J. J. Verbanc, "Recent advances in isocyanate chemistry," Chemical Reviews, vol. 57, no. 1, pp. 47-76, 1957.

[42] J. Che, Y. Xiao, X. Wang, A. Pan, W. Yuan, and X. Wu, "Grafting polymerization of polyacetal onto nano-silica surface via bridging isocyanate," Surface and Coatings Technology, vol. 201, no. 8, pp. 4578-4584, 2007.

[43] D. M. Simons and R. G. Arnold, "Relative reactivity of the isocyanate groups in toluene-2,4-diisocyanate," Journal of the American Chemical Society, vol. 78, pp. 1658-1659, 1956.

[44] M. Abrantes, A. Sakthivel, C. C. Romão, and F. E. Kühn, "A chiral menthyl cyclopentadienyl molybdenum tricarbonyl chloro complex: synthesis, heterogenization on MCM-41/MCM-48 and application in olefin epoxidation catalysis," Journal of Organometallic Chemistry, vol. 691, pp. 3137-3145, 2006.

[45] R. I. Kureshy, I. Ahmad, N.-U. H. Khan et al., "New immobilized chiral Mn(III) salen complexes on pyridine $\mathrm{N}$-oxide-modified MCM-41 as effective catalysts for epoxidation of nonfunctionalized alkenes," Journal of Catalysis, vol. 235, no. 1, pp. 28-34, 2005.

[46] M. D. Alba, Z. Luan, and J. Klinowski, “Titanosilicate mesoporous molecular sieve MCM-41: synthesis and characterization," Journal of Physical Chemistry, vol. 100, no. 6, pp. 21782182, 1996.

[47] V. Caps and S. C. Tsang, "Heterogenisation of Os species on MCM-41 structure for epoxidation of trans-stilbene," Applied Catalysis A, vol. 248, pp. 19-31, 2003.

[48] S. Shylesh and A. P. Singh, "Synthesis, characterization, and catalytic activity of vanadium- incorporated, -grafted, and immobilized mesoporous MCM-41 in the oxidation of aromatics," Journal of Catalysis, vol. 228, no. 2, pp. 333-346, 2004.

[49] A. P. Bhatt, K. Pathak, R. V. Jasra, R. I. Kureshy, N.-U. H. Khan, and S. H. R. Abdi, "Chiral lanthanum-lithiumbinaphthol complex covalently bonded to silica and MCM41 for enantioselective nitroaldol (Henry) reaction," Journal of Molecular Catalysis A, vol. 244, no. 1-2, pp. 110-117, 2006.

[50] D. Xiong, M. Chen, and M. Li, "Synthesis of parasulfonatocalix[4]arene-modified silver nanoparticles as colorimetric histidine probes," Chemical Communications, no. 7, pp. 880-882, 2008.

[51] J. Sauer, F. Marlow, and F. Schuth, "Simulation of powder diffraction patterns of modified ordered mesoporous materials," Physical Chemistry Chemical Physics, vol. 3, pp. 5579-5584, 2001.

[52] M. H. Lim and A. Stein, "Comparative studies of grafting and direct syntheses of inorganic-organic hybrid mesoporous materials," Chemistry of Materials, vol. 11, pp. 3285-3295, 1999.

[53] B. Marler, U. Oberhagemann, S. Vortmann, and H. Gies, "Influence of the sorbate type on the XRD peak intensities of loaded MCM-41," Microporous Materials, vol. 6, no. 5-6, pp. 375-383, 1996.

[54] O. Carmody, R. Frost, Y. Xi, and S. Kokot, "Surface characterisation of selected sorbent materials for common hydrocarbon fuels," Surface Science, vol. 601, no. 9, pp. 2066-2076, 2007.

[55] A. Sakthivel, A. K. Hijazi, M. Hanzlik, A. S. T. Chiang, and F. E. Kühn, "Heterogenization of $\left[\mathrm{Cu}\left(\mathrm{NCCH}_{3}\right)_{6}\right]\left[\mathrm{B}\left(\mathrm{C}_{6} \mathrm{~F}_{5}\right)_{4}\right]_{2}$ and its application in catalytic olefin aziridination," Applied Catalysis A, vol. 294, pp. 161-167, 2005. 

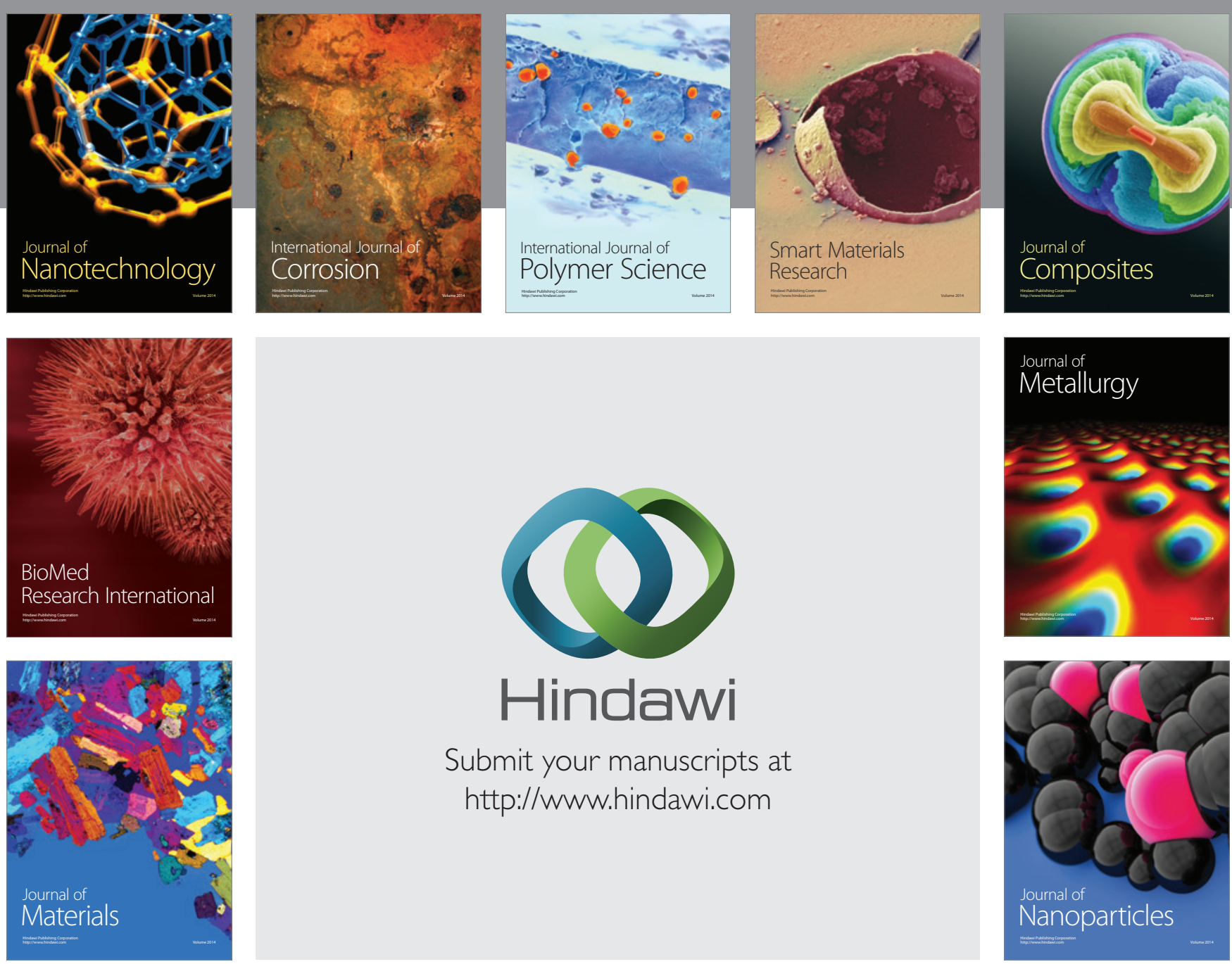

Submit your manuscripts at http://www.hindawi.com
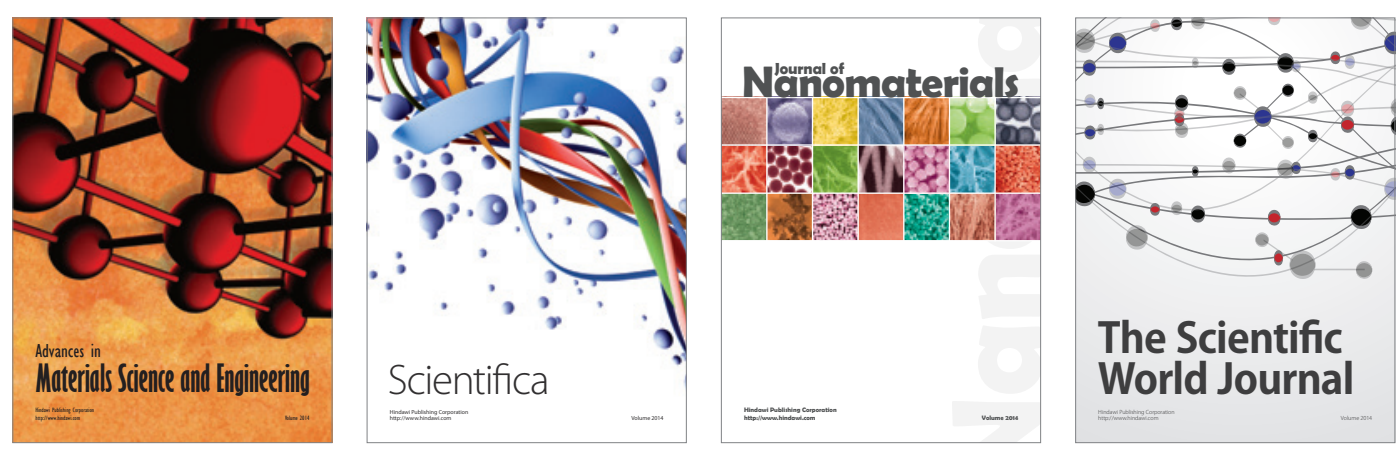

\section{The Scientific World Journal}
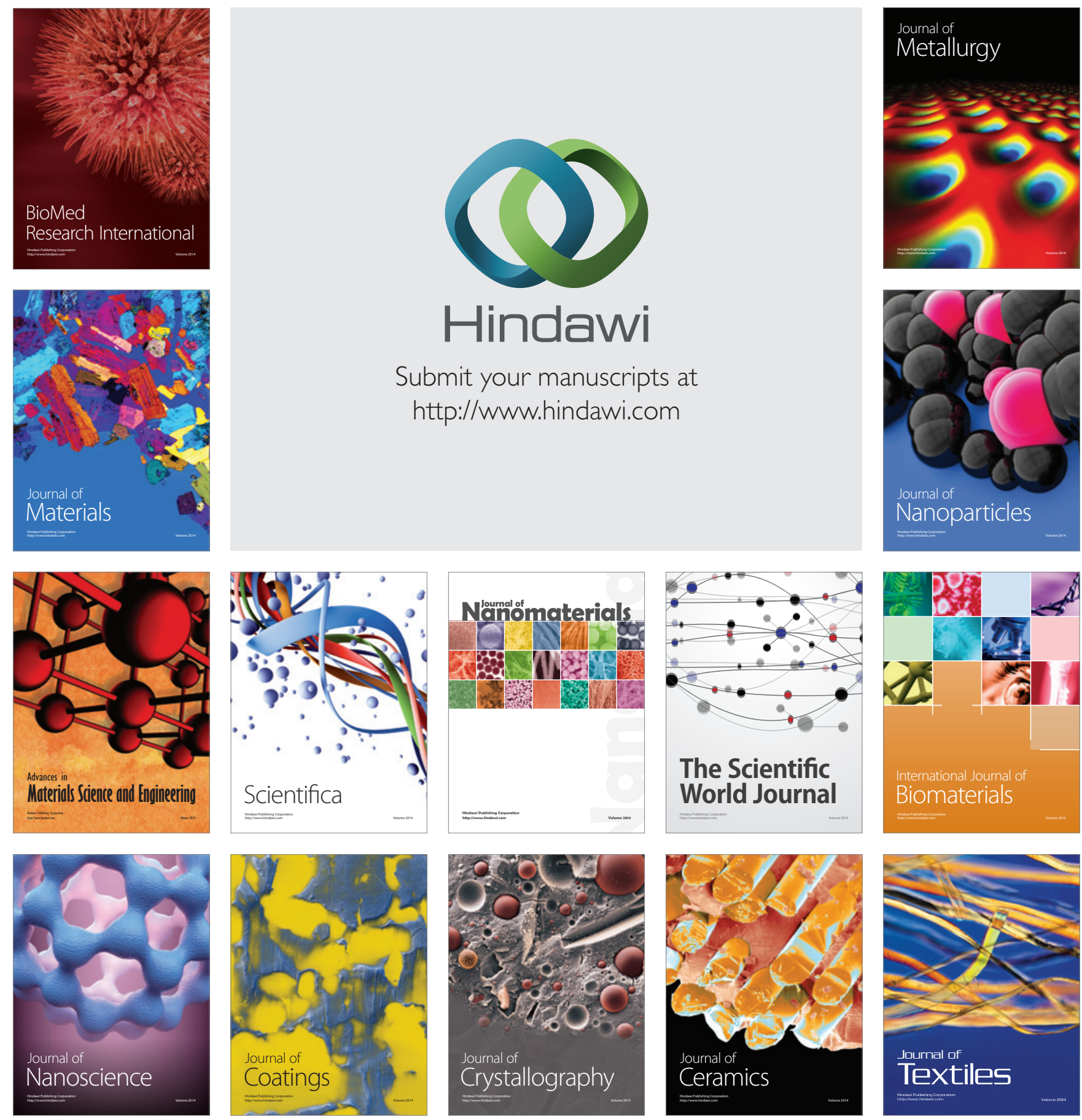